\title{
A Decade of Vector Fitting Development: Applications on Signal/Power Integrity
}

\author{
Chi-Un Lei*, Yuanzhe Wang*, Quan Chen*, and Ngai Wong* \\ * Department of Electrical and Electronic Engineering \\ The University of Hong Kong, Pokfulam Road, Hong Kong \\ Email: $\{$ culei, yzwang, quanchen, nwong \}@eee.hku.hk
}

\begin{abstract}
Vector Fitting (VF) has been introduced as a partial-fraction basis response fitting methodology for over a decade. Because of its reliability and versatility, VF has been applied and extended to a number of areas. In this book chapter, we will discuss the applications of VF in the context of macromodeling of linear structures in signal/power integrity analyses. We will also discuss main features of VF along three directions: data, algorithms and models. Two practical examples are given to demonstrate the merits of VF. An alternative $P$-norm approximation criterion is proposed to enhance the accuracy of the macromodeling process.
\end{abstract}

Keywords: Signal Integrity, Power Integrity, Vector Fitting, Macromodeling, Approximation PACS: 02.60.Cb, 02.60.Ed, 02.60.Gf, 85.40.Bh

\section{INTRODUCTION}

Vector Fitting (VF) [1] is a numerical technique for sampled response-matching system identification (macromodeling), which involves iterative linear least-squares solves with a partial fraction basis. In contrast to other system identification techniques for broadband (from DC to GHz) system identification, VF avoids ill-conditioned calculation, and therefore works in a more robust and efficient manner. Furthermore, its theoreticallysimple and versatile framework can easily incooperate various constraints by introducing a variety of extensions to many areas. VF has also been used in modeling of different electrical systems $[1,2]$ and extended to different areas, for example, filter design $[3,4,5]$, power network analysis [6, 2] and electromagnetic (EM) simulation [7, 8].

The idea of VF was firstly introduced for transmission line transient modeling in [9]. The underlying idea of VF is to replace the approximated (or initialized) poles with an improved set of poles through implicit weighting (the pole relocation technique), which thereby improves the approximation iteratively. VF approximates an underlying system to a new system using partial fraction basis with real or complex conjugate poles. A number of generalizations and extensions have been proposed for better VF performance and integration with various identification requirements. VF has been thoroughly discussed in $[10,11]$. Its variants have been widely used in industrial electronic design workflows for addressing signal integrity issues.

This paper acts as an extended tutorial on VF on top of [12]. We first give a brief introduction to the signal/power integrity issues and the basic formulation of VF. Then we discuss the applications of VF in system identification. Finally an alternative $P$-norm approximation criterion in VF is proposed for approximation enhancement, which is 


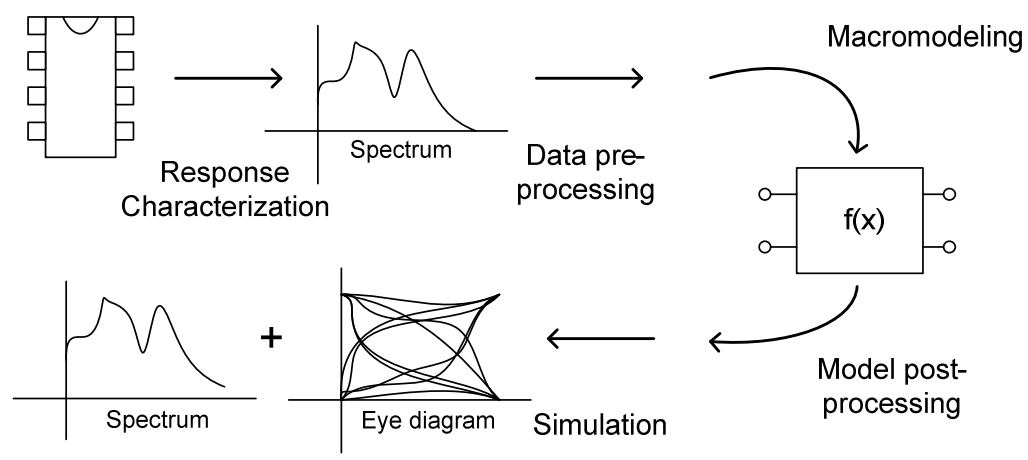

FIGURE 1. Common macromodeling flow in signal integrity analyses.

verified through numerical examples.

\section{LINEAR MACROMODELING: SYSTEM IDENTIFICATION PROBLEM IN SIGNAL/POWER INTEGRITY}

Electronic systems, such as smartphones, computers and high-definition televisions, have become essential to our daily lives. From a system perspective, these electronic systems contain modules of integrated circuits (ICs), e.g., memory, datapath, control and input-output circuitry. Modules are connected by interconnect (viz. wire) and power networks, including via holes, sockets, power/ground planes, wires, connectors and chip packages. In low-speed circuit operation, interconnect networks perform as ideal wires, which do not distort transmitted signals, and the electronic system functions well according to the functional-level design.

However, with the increasing operation frequency and decreasing feature size of ICs, high-frequency effects, such as signal delay, crosstalk, interconnect dispersion and mutual couplings, have become dominant factors limiting system performance. Therefore, signal integrity verification and design have become popular practices in the IC design process. Signal integrity analysis can be done through checking the eye diagram of transmission channels on a circuit board from measurement or simulation. Accurate and efficient modeling is required to capture high-frequency behaviors of systems for pre-layout simulation in the design phase, so as to ensure consistent signal transmissions and reliable power distributions in high-speed electronic systems [13, 2, 14]. However, a full-wave electromagnetic (EM) analysis over a global system is impractical. Reduced models with similar properties to the original systems are therefore demanded.

For structures with complicated geometry, such as packages, circuit boards and radiofrequency $(\mathrm{RF})$ objects, data-driven linear macromodeling is usually applied. A common macromodeling flow is shown in Fig. 1. The sampled structure responses can be obtained by exciting one input port at a time and computing or measuring the re- 
sponses at the output ports (Response Characterization). By approximating the sampled frequency-dependent or time-dependent system response data, a macromodel is generated to replace the original large-order system by a smaller-order one with similar input-output (I/O) behaviors (Macromodeling). The macromodel is used to produce spectra and waveforms for signal integrity analysis and/or coupled with other circuit model blocks (e.g., logic devices) for global simulation (Simulation). Peripheral preand post-processing techniques are used to modify the macromodel characteristics and enhance the simulation performance.

Generally, for a single-port system, macromodeling techniques intend to fit a linear time-invariant (LTI) system to the desired continuous-time frequency domain ( $s$-domain) response $H(s)$ at a set of calculated/sampled points at the I/O ports. The model is usually a state-space system or a rational transfer function with a set of basis $\left\{\phi_{n}\right\}$

$$
H(s) \approx \frac{N(s)}{D(s)}=\sum_{n=1}^{N} b_{n} \phi_{n}(s) / \sum_{n=1}^{N} \widetilde{b}_{n} \phi_{n}(s),
$$

where $\widetilde{b}_{n}, b_{n} \in \mathbb{R}, N$ is the macromodel order. The algorithm is usually required to fit tens of ports in the original system, where each port contains hundreds of frequency sampled data points. Therefore, the linear structure macromodeling can be classified as a largescale broadband system identification problem. The system identification process must meet several stringent constraints specific to the macromodeling process, namely, accurate and physically consistent response approximation, low computation complexity, numerically robust computation and least manual configuration during calculation.

In the $L_{2}$ sense, the optimal model of a system can be obtained through minimizing the following objective function

$$
\min \left\|\frac{N(s)}{D(s)}-H(s)\right\|_{2} .
$$

However, this is a numerically sensitive non-linear problem with no prior information about the exact pole and zero locations of the system being identified. The response is usually approximated using Prony's method [15] for a coarse solution or other identification frameworks for a finer solution, such as continuous-time domain SanathananKoerner (SK) iteration [16] or equivalent discrete-time domain Steiglitz-McBride (SM) iteration [17]. The objective function of the SK iteration in the ith iteration is

$$
\min \left\|\frac{N^{(i)}(s)}{D^{(i-1)}(s)}-\frac{D^{(i)}(s)}{D^{(i-1)}(s)} H(s)\right\|_{2} .
$$

By arranging the weighting function $\sigma^{(i)}(s):=D^{(i)}(s) / D^{(i-1)}(s)$, the model parameters can be determined via a least-squares problem

$$
\underbrace{\frac{N^{(i)}(s)}{D^{(i)}(s)} \frac{D^{(i)}(s)}{D^{(i-1)}(s)}}_{(\sigma H)^{(i)}(s)}-\underbrace{\frac{D^{(i)}(s)}{D^{(i-1)}(s)}}_{\sigma^{(i)}(s)} H(s) \approx 0 .
$$


If a monomial power series basis function is used in (4) for broadband macromodeling, i.e., $\phi_{n}(s)=s^{n}$, the traditional SK iteration approach will suffer from an ill-conditioned Vandermonde matrix calculation [18], and thereby do not satisfy the macromodeling requirements from a numerical perspective. Therefore, Vector Fitting (VF) is recognized as a robust and simple broadband macromodeling technique, and has been widely practiced. In this paper, we will discuss the macromodeling procedure development along three directions:

1. Data section $(H(s))$ : Input data choices, pre-processing of data and model;

2. Algorithms section $(H(s) \rightarrow N(s) / D(s))$ : Identification criterion and framework and numerical implementation;

3. Models section $(N(s) / D(s))$ : Post-processing for model physical consistency and simulation.

\section{FORMULATION OF VECTOR FITTING (VF)}

In $\mathrm{VF}$, given a set of poles $\left\{\alpha_{n}\right\}$, (1) is approximated using a summation of partial fraction basis and a unity basis with their model parameters $\left\{c_{n}\right\}$ and $d$,

$$
H(s) \approx \frac{N(s)}{D(s)}=\left(\sum_{n=1}^{N} \frac{c_{n}}{s+\alpha_{n}}\right)+d .
$$

By including the weighting function $\sigma(s),(5)$ is linearized into an iterative separable denominator calculation, namely, for the $t$ th iteration,

$$
\underbrace{\left(\sum_{n=1}^{N} \frac{c_{n}^{(t)}}{s+\alpha_{n}^{(t)}}\right)+d^{(t)}}_{\sigma H^{(t)}(s)} \approx \underbrace{\left(\left(\sum_{n=1}^{N} \frac{\gamma_{n}^{(t)}}{s+\alpha_{n}^{(t)}}\right)+1\right)}_{\sigma^{(t)}(s)} H(s),
$$

which falls into the framework of SK iteration (4) $[18,19]$.

In numerical implementation, provided all poles are real and $N_{s}$ frequency-sampled data points are given, an expression from (6) is formed for each frequency-sampled point $s_{i}, i=1,2, \ldots, N_{s}$,

$$
\mathbf{A}_{i} \mathbf{x}=b_{i},
$$

where $\quad b_{i}=H\left(s_{i}\right), \quad \mathbf{A}_{i}=\left[\begin{array}{lllllll}\frac{1}{s+\alpha_{1}^{(t)}} & \cdots & \frac{1}{s+\alpha_{n}^{(t)}} & 1 & \frac{-H\left(s_{i}\right)}{s+\alpha_{1}^{(t)}} & \cdots & \frac{-H\left(s_{i}\right)}{s+\alpha_{n}^{(t)}}\end{array}\right], \quad$ and $\mathbf{x}=\left[\begin{array}{lllllll}c_{1}^{(t)} & \cdots & c_{N}^{(t)} & d^{(t)} & \gamma_{1}^{(t)} & \cdots & \gamma_{N}^{(t)}\end{array}\right] . \mathbf{x}$ are solved through stacking the row (7) at the $N_{S}$ sampled points to form an overdetermined linear equations problem,

$$
\left[\begin{array}{llll}
\mathbf{A}_{1}^{T} & \mathbf{A}_{2}^{T} & \cdots & \mathbf{A}_{N_{s}}^{T}
\end{array}\right]^{T} \mathbf{x}=\left[\begin{array}{llll}
b_{1} & b_{2} & \cdots & b_{N_{s}}
\end{array}\right]^{T},
$$

where it can be solved through normal equations or a $\mathrm{QR}$ decomposition. The zeros of $\sigma^{(t)}(s)$ (i.e., the new set of poles $\left\{\alpha_{n}^{(t+1)}\right\}$ ) can be calculated as the eigenvalues of the 
matrix

$$
\Psi=\left[\begin{array}{cccc}
\alpha_{1}^{(t)} & & & \\
& \alpha_{2}^{(t)} & & \\
& & \ddots & \\
& & & \alpha_{N}^{(t)}
\end{array}\right]-\left[\begin{array}{c}
1 \\
1 \\
\vdots \\
1
\end{array}\right]\left[\begin{array}{c}
\gamma_{1}^{(t)} \\
\gamma_{2}^{(t)} \\
\vdots \\
\gamma_{N}^{(t)}
\end{array}\right]^{T}
$$

If the poles are unstable (i.e., $\Re\left(\left\{\alpha_{n}^{(t+1)}\right\}\right)>0$ ), the poles are flipped against the imaginary axis to the open left half plane for pole stabilization

$$
\alpha_{n}^{(t+1)}:=-\alpha_{n}^{(t+1)} \text {. }
$$

This is equivalent to cascading an allpass filter $A(s)$ to alter the phase response

$$
A(s)=\frac{s+\alpha}{s-\alpha} \text {. }
$$

The computation is repeated until convergence is achieved, say, $\sigma(s) \approx 1$ and $\left\|\frac{N^{(t)}(s)}{D^{(t)}(s)}-H(s)\right\| \approx 0$, at the $N_{T}$ th iterations. Eq. (6) is then reduced to

$$
\sum_{n=1}^{N} \frac{c_{n}^{\left(N_{T}\right)}}{s+\alpha_{n}^{\left(N_{T}\right)}}+d^{\left(N_{T}\right)} \approx H(s),
$$

and the residues $\left\{c_{n}^{\left(N_{T}\right)}\right\}$ and $d^{\left\{N_{T}\right\}}$ can be calculated similarly as in (8). In summary, VF replaces the monomial power series basis by a partial fraction basis, which significantly improves the numerical condition in calculation of (8). The detailed VF formulation is shown in $[1,18,19]$. Pseudocodes are given to summarize the framework of VF:

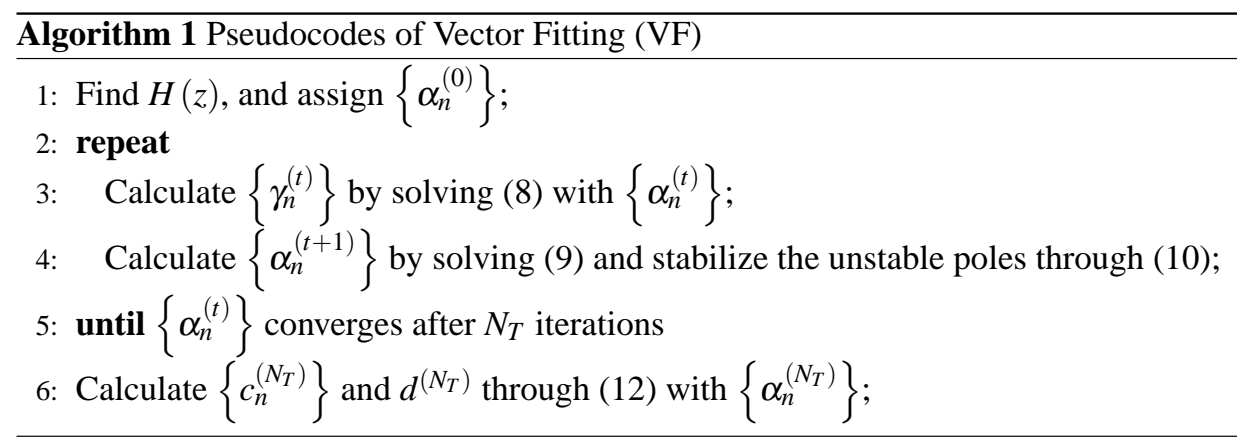

\section{DATA}

Data describe the system response, and are obtained from measurements or EM simulators. Since data content can affect the properties and quality of the macromodel, different considerations and techniques have been proposed to ensure the input data are maximally informative for identification purpose. 


\section{Data: Input Data Choices}

Continuous-time frequency-sampled data $H(s)$ are used for macromodeling in VF [1], as the frequency-sampled responses capture the high-frequency behaviors of a system. Examples of frequency-sampled data are scattering parameters ( $S$-parameters) for RF objects and admittance parameters ( $Y$-parameters) for interconnects. Alternative data choices, such as frequency response derivative $H^{\prime}(s)$ [20], phase response $\angle H(s)$ [21] and magnitude response $|H(s)|$ [22], are used for different identification purposes. In practices, frequency-domain macromodeling involves complicated measurements. Truncated time-sampled data (input and output response $X[n]$ and $Y[n]$ ) are often used, therefore (discrete) time-domain VF have been proposed [23, 24]. Approximation using combination of several classes of data (hybrid-domain approximation) provides extra information for a more accurate approximation of the system. It has been applied to digital IIR filter approximation [4] and works well in macromodeling process.

\section{Data: Pre-Processing of Data}

The system response should correctly describe the system. However, some problems, such as data burst, defects, missing and noise-disturbance, may occur during the course of data collection. Some information may get lost and difficulties and failures in approximation may arise. Therefore, data pre-processing is required to ensure the data are meaningful (e.g., passive and causal) to generate a correct macromodel. For example, causality and passivity verifications of input data and delay extraction are developed using (generalized) Hilbert transform [25]. Furthermore, causality-constrained data interpolation is developed to generate consistent DC and low-frequency data, which is necessary for simulation but usually not provided in the frequency-sampled data [25].

In addition, a large data set or broadband responses usually have a large variance and may result in ill-conditioned calculation. Pre-filtering techniques, in this scenario, can be used to change the distribution of noise and bias, so as to give a better fitting of important frequency range and a numerically favorable calculation with small computational cost. An appropriate adaptive or deterministic data selection process and response weighting can also help improve the approximation.

\section{Data: Pre-Processing of Model}

A priori configuration of macromodels should be chosen based on the knowledge of the algorithms (SK iteration) and data for a convenient approximation. For example, an a priori model order selection helps generate a minimum size macromodel for efficient simulations with accuracy control. The model order can be selected by applying experimental observation of the frequency response in frequency-sampled data [26], or the Hankel Singular Value (HSV) in (discrete) time-sampled data [24]. 


\begin{abstract}
ALGORITHMS
Given a set of input data, an algorithm is used to determine the model parameters. A good algorithm should have an appropriate identification criterion and should be easy and robust for numerical implementation. We first discuss the algebraical minimization criteria, then the numerical implementation for a numerically favorable model parameters calculation.
\end{abstract}

\title{
Algorithms: Identification Criterion and Framework
}

The selection of approximation criteria is important for model approximation. The approximated model should be reliable, obtained within a reasonable computation time, and should admit an exact description of the true system. SK iteration with an $L_{2}$-norm prediction error is usually used since it is applicable to different response models. Other criterion extensions are also developed recently for specific applications [27].

Massive-port macromodeling: VF handles multi-port macromodeling by stacking the system equation matrices of responses of all ports into a single column of over-determined equation for solutions. However, numerical difficulties exist in modeling the systems with a large number of ports (e.g., package parasitic networks and electromagnetic-aware circuits). To model a system with an arbitrary number of ports, a reformation of the VF framework is proposed to approximate the eigenpairs rather than the matrix elements [28]. It gives a more accurate approximation for systems with a large ratio between the largest and smallest eigenvalues.

Parametric macromodeling: Variabilities in geometry and material properties are generated during the manufacturing process, and become a critical factor in nano-scale high-frequency circuit simulation and design. In order to accurately predict the behavior and reduce the computation time of repeated simulations, a parametric macromodel is used to describe the variational structures

$$
H(s, g) \approx \frac{\sum_{n=0}^{N_{s}}\left(\sum_{p=1}^{P} b_{n p} \varphi_{p}(g)\right) \phi_{n}(s)}{\sum_{n=0}^{N_{s}}\left(\sum_{p=1}^{P} \widetilde{b}_{n p} \varphi_{p}(g)\right) \phi_{n}(s)},
$$

where $\phi_{n}(s)$ is the frequency-dependent basis and $\varphi_{p}(g)$ is the variability-dependent basis with a single variational parameter $g$ and $P$ samples in the variability domain. The variational structures can be described by a macromodel with a polynomial basis or rational function basis $[29,30,11]$.

\section{Algorithms: Numerical Implementation}

Due to the nature of iterative calculation, its implementation is usually numerically sensitive. Although VF solves the ill-conditioned calculation by a partial-fraction basis, some other problems, such as inappropriate initial guess and noise-contaminated responses, tend to damage the algorithm convergence. Some improvements have been proposed to address these problems. 
Initial poles and applied basis: The algorithm gives a set of model parameters $\left(b_{n}\right.$ and $\tilde{b}_{n}$ in (1)) according to the given set of basis $(\phi(s))$, the sampled data and the initial poles. The selected basis affects the conditioning of the system equation matrix in (8) and the solution accuracy.

One approach to address this problem is to select an appropriate set of initial poles. The initial poles can be obtained by a simple calculation (e.g., Prony method [15]), or intuitively assigned as a set of weakly-damped initial poles $\left(\alpha_{1,2}=a \pm j 0.01 a\right)$ [1]. Another approach is to select a robust basis for calculation, which minimizes the numerical disturbance due to the inappropriate set of poles. Orthonormal basis $\phi_{o r_{-} n}(s)[31]$ and discrete-time domain (z-domain) basis $\phi_{z n n}(z)$ [3] have been proposed based on this idea, namely,

$$
\begin{gathered}
\phi_{o r \_n}(s)=\kappa_{n} \sqrt{2 \Re\left(\alpha_{n}\right)}\left(\prod_{j=1}^{n-1} \frac{s-\alpha_{j}^{*}}{s+\alpha_{j}}\right) \frac{1}{s+\alpha_{n}}, \\
\phi_{z_{-} n}(z)=\frac{1}{z^{-1}+\alpha_{n}},
\end{gathered}
$$

where $\kappa_{n}$ is the normalization coefficient and $*$ denotes complex conjugate. Orthonormal basis, from a mathematical perspective reduces the condition number of the system equation matrix, while the discrete-time basis calculation maps the left Laplace plane to a unit circle plane, and thus improves the numerical condition from a signal-processing perspective. Furthermore, discrete-time domain orthonormal basis is proposed recently for further robustness improvement [32]. Other basis generalizations are also available for different requirements, e.g., modeling the responses with repeated poles [31].

Macromodeling with noisy signals: Experiences show that the convergence is slowed down in noise-contaminated signals and biased in the low-frequency region. This is because the unity basis of $\sigma(s)$ in (6) impairs the LS normalization of equation solving. To address this problem, a variable unity basis $\left(\gamma_{0}\right)$ normalization (16) with an additional relaxed nontriviality condition (17) is adopted for a relaxed least-squares normalization (Relaxed VF) [33, 19],

$$
\begin{gathered}
\underbrace{\left(\sum_{n=1}^{N} \frac{c_{n}^{(t)}}{s+\alpha_{n}^{(t)}}\right)+d_{n}^{(t)}}_{(\sigma H)^{(t)}(s)} \approx \underbrace{\left(\left(\sum_{n=1}^{N} \frac{\gamma_{n}^{(t)}}{s+\alpha_{n}^{(t)}}\right)+\gamma_{0}\right)}_{\sigma^{(t)}(s)} H(s), \\
\Re\left(\sum_{k=1}^{N_{s}}\left(\sum_{n=1}^{N}\left(\gamma_{n} \phi_{n}\left(s_{k}\right)\right)+\gamma_{0}\right)\right)=N_{s}+1 .
\end{gathered}
$$

Eq. (17) imposes that the sum of the samples approaches to a nonzero value. This improves the normalization of the transfer function coefficients and the linearization of the iterative SK iteration without affecting the convergence.

Massive-port macromodeling: VF suffers from computational inefficiency when macromodeling massive-port systems due to the unnecessary calculation of $c_{n}$ in (8) during iterative pole calculation (Step 3). Based on the observation of shared common 
poles in the macromodel, a QR decomposition is applied to extract the calculation of $\gamma_{n}$ of each port response and formulate a compacted calculation [34]. The computational complexity is then reduced from $O\left(\left(P_{\text {in }} P_{\text {out }}+1\right)^{2} n^{2} N_{s} P_{\text {in }} P_{\text {out }}\right)$ to $O\left(n^{2} N_{s} P_{\text {in }} P_{\text {out }}\right)$ for a system with $P_{\text {in }}$ input ports and $P_{\text {out }}$ output ports, without any lost of accuracy.

\section{MODELS}

The macromodel (model) describes the Input-Output (I/O) characteristics of the approximated system, for analysis and simulation with other circuit models. The model should be accurate, physically consistent and of low complexity for simulation. Necessary postprocessing techniques are adopted to ensure a correct simulation.

\section{Models: Post-Processing for a Physically Consistent Model}

The macromodel should be physically consistent, i.e., real-valued, stable, passive and causal [35].

Real-valued: Real-valued macromodels do not generate complex-valued responses for real-valued input data. However, the original VF may generate complex-valued macromodels if the complex poles are not restricted to conjugate pairs. Some modifications in (7)-(9) are required to construct a real-valued macromodel, as explained in [1]. Complex-valued computations of (8) are separated into its real and imaginary parts to avoid numerical errors, at the expense of an increased problem size.

Stable: Stable macromodels do not generate response beyond limits for any bounded input signal. An unstable pole can be stabilized through a non-linear pole flipping in (11). The flipping, however, does not affect the norm criterion in (3) and the algorithm convergence.

Passive: Passive macromodels do not generate energy, yet VF may generate slightly non-passive macromodels due to numerical errors. Therefore, passivity enforcement through perturbation of model parameters is required to passify the model, and a detailed study is shown in [36].

Causal: Causal macromodels do not generate output signal according to the future input. However, modeling electrically-long structures (i.e., responses with a signal delay) using a purely rational macromodel may suffers from inapplicable fitting and often generates a non-causal model. A reformulated VF is developed [37]. With the $D$ obtained time delays $\left\{\tau_{d}\right\}$, the response can be fitted via

$$
H(s) \approx \frac{\sum_{n=0}^{N} \sum_{d=1}^{D} b_{n d} \phi_{n}(s) e^{-s \tau_{d}}}{\sum_{n=0}^{N} \widetilde{b}_{n d} \phi_{n}(s)} .
$$

\section{Models: Post-Processing for Simulation}

The approximant macromodel is used to generate the frequency response, timedomain reflectometry (TDR) waveforms, time-domain transmissometry (TDT) wave- 
(a)

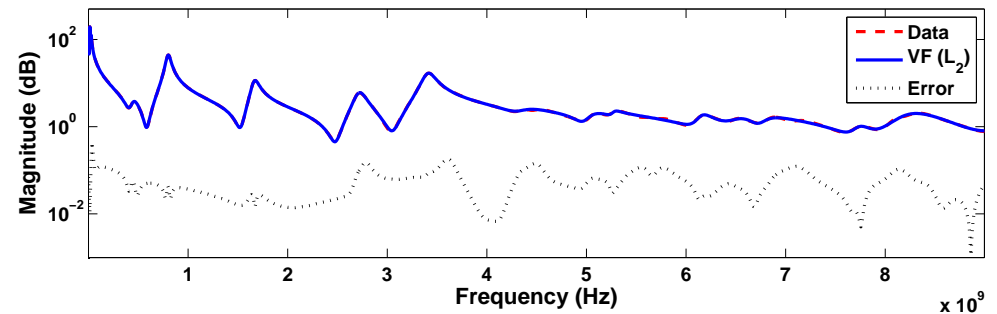

(b)

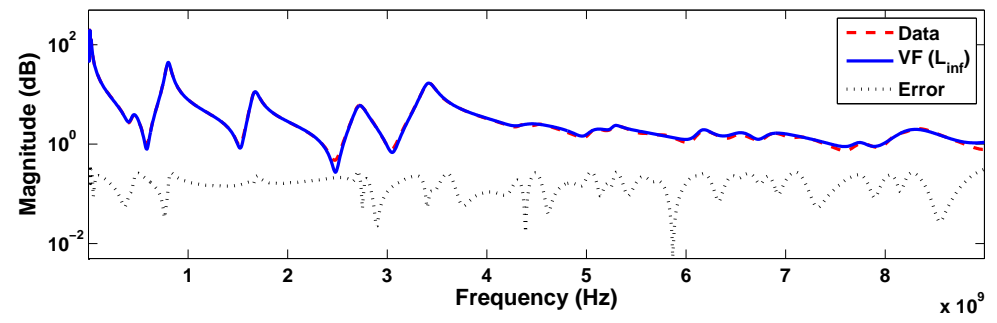

FIGURE 2. Magnitude responses of the power distribution network: (a) approximation using $L_{2}$ norm, and (b) approximation using $L_{\text {inf }}\left(L_{\infty}\right)$ norm.

(a)

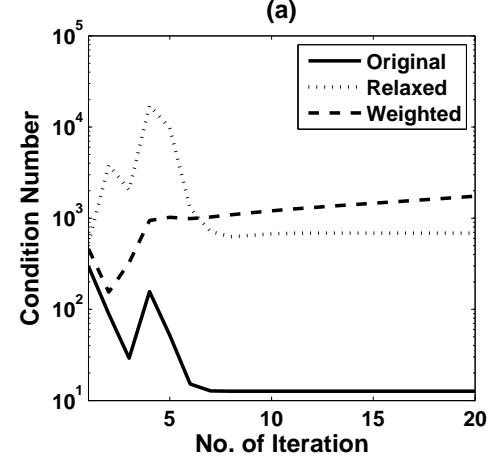

(b)

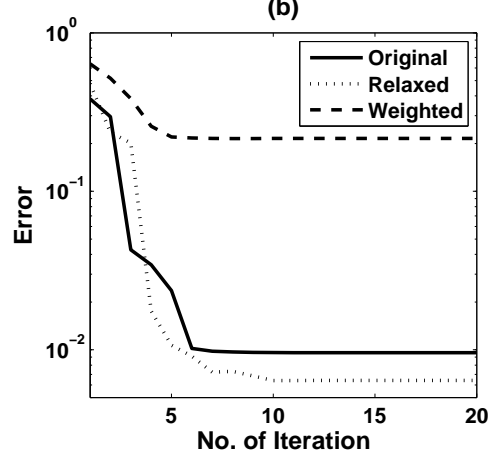

FIGURE 3. (a) Condition number of the system equation matrix in (8), and (b) $L_{2}$ error of the approximation using original VF (6), relaxed VF (16) and weighted VF.

forms and eye diagrams for channel analysis, or coupled with other models for overall simulation. Therefore, the models should be fully integrated with simulation tools for efficient analysis. The macromodel can be described by a pole-residue form in Matlab Simulink or Verilog-A description for high-level simulations. The macromodel can also be described as an equivalent circuit in a SPICE netlist for co-simulation with other (non-linear) macromodels [38]. A standard equivalent circuit can be generated using differential-equation realization. 


\section{$P$-NORM APPROXIMATION IN VF}

To satisfy different macromodeling requirements and give a more realistic description of the system, the approximation framework (3) is extended to a $P$-norm $\left(L_{p}\right)$ approximation. The minimization framework (3) is generalized to

$$
\min \left\|\frac{N^{(t)}(s)}{D^{(t-1)}(s)}-\frac{D^{(t)}(s)}{D^{(t-1)}(s)} H(s)\right\|_{p},
$$

for which the over-determined equations can be efficiently solved by convex programming. The approximation framework can be generalized to a user-defined norm (e.g., region-dependent norm) approximation or (norm-)constrained approximation to meet different macromodeling requirements. For example, $L_{\infty}$ (Chebyshev norm) approximation gives a smaller macromodel for a linear-phase (time-delayed) response, $L_{2}$ approximation gives a more accurate macromodel for a noisy response, and $L_{1}$ approximation is favorable for system identification with an impulsive-noise-contaminated signal.

\section{NUMERICAL EXAMPLES AND DISCUSSIONS}

The VF is coded in Matlab m-script files and run in the Matlab 7.5 on a 1GB-RAM $3.4 \mathrm{GHz}$ PC. The first example arises from a power distribution network of an IC power plane [2], whose admittance responses range from $\mathrm{DC}$ to $9 \mathrm{GHz}$. The port response is fitted using relaxed VF [33] with a 35th-order macromodel with 10 iterations (18.28 seconds) and a set of linear-spaced initial poles, which gives $0.0064 L_{2}$ and $0.0022 L_{\infty}$ error in fitting. Fig. 2 plots the magnitude-domain responses of the converged approximant. Fig. 3 shows the condition number of the system equation matrix (8) and the $L_{2}$ error during iterations. In general, VF converges quickly ( $\leq 10$ iterations), especially for minimum-phase (passive) response. For further analysis of generalizations of VF, we repeat the example using VF without relaxed constraint and relaxed VF with a inversemagnitude weighting. The quantitative comparison is shown in Fig. 3. It shows that the weighting does not contribute much to the numerical condition, but it affects the convergence. The relaxation may affect the numerical condition of the calculation, but it also significantly improves the accuracy of the approximation. At last, we repeat the example under an SNR of $-35 \mathrm{~dB}$. In this case, relaxed VF converges with $0.0193 L_{2}$ and 0.0014 $L_{\infty}$ error. This shows the relaxed VF is robust to the noisy response approximation.

The responses are also fitted using $L_{\infty}$ norm approximation with the same configuration and clean signal, which gives an approximation with $0.0165 L_{2}$ error and $0.0016 L_{\infty}$ error. The magnitude-domain response of the converged approximation in Fig. 2 shows that $L_{\infty}$-norm approximation renders a more accurate low-frequency (near DC) approximation which is important for simulation, and $P$-norm approximation can be used as an alternative approximation criterion.

The performance of VF is also verified by a large macromodeling example of a fourteen-port power distribution network of a communication board. The tested intelligent network communicator (INC) board contains digital, ratio frequency (RF) and optoelectronic sections on a single $83 \mathrm{~cm} \times 65 \mathrm{~cm}$ test bed. The board has two FPGA 


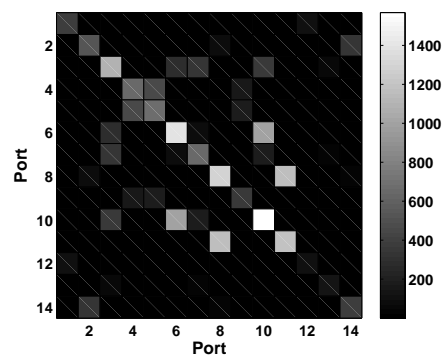

FIGURE 4. Signal energy distribution of the power distribution network system.

(a)

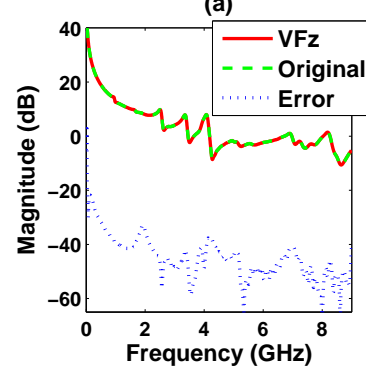

(b)

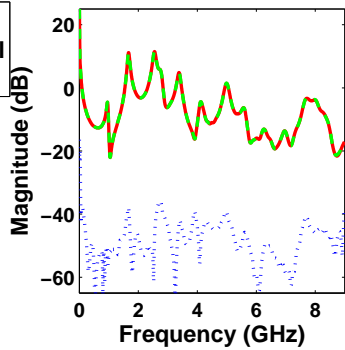

(c)

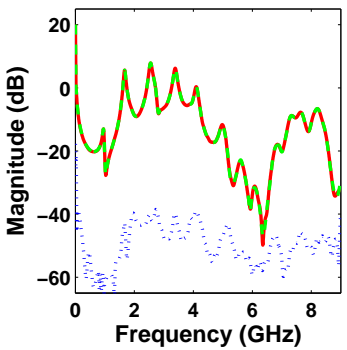

FIGURE 5. Magnitude responses of the power distribution network macromodeling: (a) Port $(6,10)$, (b) Port $(7,13)$, and (c) Port $(10,13)$.

chips, one multiplexer (MUX), one RF amplifier, one signal layer, one irregularly shaped ground layer and one irregularly shaped power plane layer. $14 \times 14$ admittance parameter matrix is generated at 1286 frequencies ranging from $10 \mathrm{KHz}$ to $9 \mathrm{GHz}$ [39]. Fig. 4 plots the signal energy distribution of the approximated system, from which we can see that some pairs of ports are not coupled with each other. By extracting the coupled responses from all the responses, the approximation is significantly reduced from fitting 196 responses to fitting 54 responses. The frequency-sampled responses are fitted using VF with a 100-pole approximant. It takes VF 99 seconds and 5 iterations for VF to converge, with the average relative error being 0.0096. Fig. 5 plots some typical approximations. Fig. 6 plots the distribution of the relative error of energy. This example has a large amount of response data to be fitted, but VF is computationally well-conditioned and gives an accurate approximation within a few iterations.

Some discussions about the macromodeling process are in order:

1. The computation time is exponentially proportional to the amount of fitting data, which depends on the number of ports and the number of samples.

2. The computational difficulties are related to the characteristics of the underlying structure and sampling distributions. In some situations, the approximation accuracy does not get improved much with higher order models or more iterations, which implies some generalized models (e.g., grey-box models) are required to bet- 


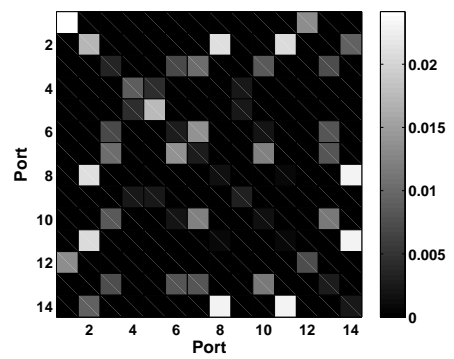

FIGURE 6. Relative error energy distribution of the power distribution network macromodel.

ter describe the system. Some prior application-specific configurations can be applied based on physical insights of the measured data. For example, pre-processing techniques (e.g., frequency warping or delay extraction) or delay-based macromodeling techniques are used to model delayed responses. Furthermore, it is not feasible to model multi-pole structures (e.g., RF circuits) by single-pole partial fraction basis, in which case multi-pole basis are required for approximation [31]. Automatic a priori response characterization procedures and specialized models should be developed to facilitate the macromodeling process.

3. Measured responses are generally more difficult to model compared to simulated responses. It is because measured responses have irregular disturbances, which affect the convergence and accuracy of iterative numerical calculation frameworks. On the other hand, the amount of fitting data is not necessarily related to the approximation accuracy.

4. In addition to the effort to improve its accuracy and speed, we should also make sure that the macromodeling algorithm requires least manual configurations. For example, the order of the model, the locations of initial poles, the number of iterations and the criterion of approximation should be automatically determined instead of manually selected.

5. An alternative choice of macromodeling process is the Loewner matrix-based tangential interpolation algorithm [40, 41], which is a non-iterative and manual configurations free macromodeling method using frequency-sampled responses. The order of the underlying system is also automatically recognized.

\section{CONCLUSIONS}

By applying a partial fraction basis, Vector Fitting (VF) has demonstrated its numerical robustness in broadband system identification. The good performance and versatile extensibility of VF render it an attractive tool for signal/power integrity analyses. In this chapter, different issues related to VF have been discussed on how to obtain a good macromodel for simulation. Furthermore, a $P$-norm approximation criterion is proposed to provide an alternative measure to meet different requirements. 


\section{ACKNOWLEDGMENTS}

The work was supported in part by the Hong Kong Research Grants Council (HKU 718509E and HKU 717407E) and the University Research Committee of The University of Hong Kong.

\section{REFERENCES}

1. B. Gustavsen, and A. Semlyen, "Rational Approximation of Frequency Domain Responses By Vector Fitting," in IEEE Trans. Power Delivery, 1999, vol. 14, no. 3, pp. 1052-1061.

2. M. Swaminathan, and A. E. Engin, Power Integrity Modeling and Design for Semiconductors and Systems, Prentice Hall PTR, 2007.

3. N. Wong, and C. U. Lei, "IIR Approximation of FIR Filters Via Discrete-Time Vector Fitting," in IEEE Trans. Signal Process., 2008, vol. 56, no. 3, pp. 1296-1302.

4. C. U. Lei, and N. Wong, "IIR Approximation of FIR Filters Via Discrete-Time Hybrid-Domain Vector Fitting," in IEEE Signal Process. Lett., 2009, vol. 16, no. 6, pp. 533-537.

5. C. U. Lei, C. M. Cheung, H. K. Kwan, and N. Wong, "Efficient Design of Arbitrary Complex Response Continuous-Time IIR Filter," in Trends in Communication Technologies and Engineering Science, Springer, 2009, pp. 163-176.

6. W. Yu et .al, "Efficient Power Network Analysis Considering Multidomain Clock Gating," in IEEE Trans. Comput.-Aided Design Integr. Circuits Syst., 2009, vol. 28, no. 9, pp. 1348-1358.

7. Y. Cai, and C. Mias, "Faster 3D Finite Element Time Domain - Floquet Absorbing Boundary Condition Modelling Using Recursive Convolution And Vector Fitting," in IET Microwaves, Antennas and Propagation, 2009, vol. 3, no. 2, pp. 310-324.

8. D. Deschrijver, D. Pissoort, and T. Dhaene, "Adaptive Time-Domain Macromodeling Algorithm For Fast Termination of FDTD Simulations", in Proc. IEEE Workshop on Signal Propagation on Interconnects, 2010.

9. B. Gustavsen, and A. Semlyen, "Simulation of Transmission Line Transients Using Vector Fitting And Modal Decomposition", in IEEE Trans. Power Delivery, 1998, vol. 13, no. 2, pp. 605-614.

10. D. Deschrijver, Broadband Macromodeling of Linear Systems by Vector Fitting, PhD dissertation, University of Antwerp, Belgium (2008).

11. P. Triverio, Self Consistent, Efficient and Parametric Macromodels for High-speed Interconnects Design, PhD dissertation, Politecnico di Torino, Italy (2009).

12. C. U. Lei, Y. Wang, Q. Chen and N. Wong, "On Vector Fitting Methods in Signal/Power Integrity Applications," in Lecture Notes in Engineering and Computer Science: Proceedings of The International MultiConference of Engineers and Computer Scientists 2010, IMECS 2010, 17-19 March, 2010, Hong Kong, pp. 1407-1412.

13. R. Achar, and M. S. Nakhla, "Simulation of High-Speed Interconnects ," in Proc. IEEE, 2001, vol. 89, no. 5, pp. 693-728.

14. M. Swaminathan et .al, "Designing and Modeling for Power Integrity," in IEEE Trans. Electromagn. Compat., 2010, vol. 52, no. 2, pp. 288-310.

15. R. Kumaresan, "Identification of Rational Transfer Function From Frequency Response Sample," in IEEE Trans. Aerosp. Electron. Syst., 1990, vol. 26, no. 6, pp. 925-934.

16. C. Sanathanan, and J. Koerner, "Transfer Function Synthesis As A Ratio of Two Complex Polynomials," in IEEE Trans. Autom. Control, 1963, vol. 8, no. 1, pp. 56-58.

17. K. Steiglitz, and L. E. McBride, "A Technique for the Identification of Linear Systems," in IEEE Trans. Autom. Control, 1965, vol. 10, no. 4, pp. 461-464.

18. D. Deschrijver, B. Haegeman, and T. Dhaene, "Orthonormal Vector Fitting: A Robust Macromodeling Tool for Rational Approximation of Frequency Domain Responses," in IEEE Trans. Adv. Packag., 2007, vol. 30, no. 2, pp. 216-225.

19. D. Deschrijver, B. Gustavsen, and T. Dhaene, "Advancements in Iterative Methods for Rational Approximation in the Frequency Domain", in IEEE Trans. Power Delivery, 2007, vol. 22, no. 3, pp. $1633-1642$. 
20. T. Dhaene, and D. Deschrijver, "Generalised Vector Fitting Algorithm for Macromodelling of Passive Electronic Components," in IEE Electronics Letters, 2005, vol. 41, no. 6, pp. 299-300.

21. L. D. Tommasi, D. Deschrijver, and T. Dhaene, "Transfer Function Identification from Phase Response Data," in AEU International Journal of Electronics and Communications, 2010, vol. 64, no. 3, pp. 218-223.

22. W. Hendrickx, D. Deschrijver, L. Knockaert, and T. Dhaene, "Magnitude Vector Fitting to Interval Data," in Mathematics and Computers in Simulation, 2009, vol. 80, no. 3, pp. 572-580.

23. S. Grivet-Talocia, "Package Macromodeling Via Time-Domain Vector Fitting," in IEEE Microwave Guided Wave Lett., 2003, vol. 13, no. 11, pp. 472-474.

24. C. U. Lei, and N. Wong, "Efficient Linear Macromodeling via Discrete-Time Time-Domain Vector Fitting," in Proc. Intl. Conf. on VLSI Design, 2008, pp. 469-474.

25. P. Triverio, and S. Grivet-Talocia, "Robust Causality Characterization via Generalized Dispersion Relations," in IEEE Trans. Adv. Packag., 2008, vol. 31, no. 3, pp. 579-593.

26. N. Stevens, D. Deschrijver, and T. Dhaene, "Fast Automatic Order Estimation of Rational Macromodels for Signal Integrity Analysis," in Proc. IEEE Workshop on Signal Propagation on Interconnects, 2007, pp. 89-92.

27. F. Ferranti, Y. Rolain, L. Knockaert, and T. Dhaene, "Variance Weighted Vector Fitting for Noisy Frequency Responses," in IEEE Microwave Guided Wave Lett., 2010, vol. 20, no. 4, pp. 187-189.

28. B. Gustavsen, and C. Heitz, "Modal Vector Fitting: A Tool For Generating Rational Models of High Accuracy With Arbitrary Terminal Conditions," in IEEE Trans. Adv. Packag., 2008, vol. 31, no. 4, pp. 664-672.

29. P. Triverio, S. Grivet-Talocia, and M. S. Nakhla, "A Parameterized Macromodeling Strategy With Uniform Stability Test," in IEEE Trans. Adv. Packag., 2009, vol. 32, no. 1, pp. 205-215.

30. R. Chakraborty, A. V. Sathanur, and V. Jandhyala, "Active-Passive Co-Synthesis of Multi-GigaHertz Radio Frequency Circuits with Broadband Parametric Macromodels of On-Chip Passives," in Proc. Intl. Conf. Computer Aided Design, 2009, pp. 759-766.

31. D. Deschrijver, and T. Dhaene, "A Note On The Multiplicity of Poles In The Vector Fitting Macromodeling Method," in IEEE Trans. Microw. Theory Tech., 2007, vol. 55, no. 10, pp. 736-741.

32. B. Nouri, R. Achar, and M. S. Nakhla, " $z$-Domain Orthonormal Basis Functions for Physical System Identifications," in IEEE Trans. Adv. Packag., 2010, vol. 33, no. 1, pp. 293-307.

33. B. Gustavsen, "Improving The Pole Relocating Properties of Vector Fitting," in IEEE Trans. Power Delivery, 2006, vol. 21, no. 3, pp. 1587-1592.

34. D. Deschrijver, M. Mrozowski, T. Dhaene, and D. D. Zutter, "Macromodeling of Multiport Systems Using a Fast Implementation of the Vector Fitting Method," in IEEE Microw. Wireless Compon. Lett., 2008 , vol. 18 , no. 6 , pp. $383-385$.

35. P. Triverio, S. Grivet-Talocia, M. S. Nakhla, F. Canavero, and R. Achar, "Stability, Causality, and Passivity in Electrical Interconnect Models," in IEEE Trans. Adv. Packag., 2007, vol. 30, no. 4, pp. 795-808.

36. S. Grivet-Talocia, and A. Ubolli, "A Comparative Study of Passivity Enforcement Schemes for Linear Lumped Macromodels," in IEEE Trans. Adv. Packag., 2008, vol. 31, no. 4, pp. 673-683.

37. A. Chinea, P. Triverio, and G. Grivet-Talocia, "Delay-Based Macromodeling of Long Interconnects From Frequency-Domain Terminal Responses," in IEEE Trans. Adv. Packag., 2010, vol. 33, no. 1, pp. 246-256.

38. T. Palenius, and J. Roos, "Comparison Of Reduced-Order Interconnect Macromodels For TimeDomain Simulation," in IEEE Trans. Microw. Theory Tech., 2004, vol. 52, no. 9, pp. 2240-2250.

39. S. H. Min, Automated Construction of Macromodels from Frequency Data for Simulation of Distributed Interconnect Networks, PhD dissertation, Georgia Institute of Technology, U.S. (2004).

40. S. Lefteriu, and A. Antoulas, "A New Approach to Modeling Multi-Port Systems From Frequency Domain Data," in IEEE Trans. Comput.-Aided Design Integr. Circuits Syst., 2010, vol. 29, no. 1, pp. $14-27$.

41. Y. Wang, C. U. Lei, G. K. H. Pang, and N. Wong, "MFTI: Matrix-Format Tangential Interpolation For Modeling Multi-Port Systems," in Proc. IEEE/ACM Design Automation Conference, 2010, pp. 683-686. 
Copyright of AIP Conference Proceedings is the property of American Institute of Physics and its content may not be copied or emailed to multiple sites or posted to a listserv without the copyright holder's express written permission. However, users may print, download, or email articles for individual use. 